\title{
Understanding biobanking: An assessment of the public engagement speaking book intervention Biobanking and $M e$
}

\author{
A Bedeker, ${ }^{1}$ BA, BA (Hons), BPsych; D Anderson, ${ }^{1}$ BSc, BSc(Hons), MSc, PhD; T Lose, ${ }^{1}$ BSc, BSc (Hons), MSc; Y Mgwatyu, ${ }^{1}$ ND, BTech, MSc \\ R Luus, ${ }^{2}$ BCom, BCom (Hons), MCom, PhD; R Blignaut, ${ }^{2}$ BSc, BSc (Hons), MSc, PhD; A Christoffels, ${ }^{1}$ BSc, BSc (Hons), MScMedSc, PhD \\ 'South African National Bioinformatics Institute, South African MRC Bioinformatics Unit, University of the Western Cape, Cape Town, South Africa \\ 2 Department of Statistics and Population Studies, Faculty of Natural Sciences, University of the Western Cape, Cape Town, South Africa
}

Corresponding author: A Christoffels (alan@sanbi.ac.za)

\begin{abstract}
Background. Meaningful engagement with donors of biological samples intended for human-sample biobanking increases knowledge, and allows donors to make informed decisions. Informed donors provide a platform for public awareness, allowing communities to understand the impact of research, and may even encourage more people to donate biological samples.

Objectives. The Biobanking and Me speaking book was written to explain relevant concepts, including biobanks, genetic research and participant rights, to persons from any educational level or background. Two bilingual versions of the speaking book (English-Afrikaans and English-isiXhosa) were produced, and the impact of the speaking book was assessed.

Methods. Study participants were recruited from non-academic staffing sectors at the University of the Western Cape, South Africa. An experimental research design was followed to assess the efficacy of the speaking book in communicating the topics covered. Paired $t$-tests were conducted to compare pre- and post-test scores. Descriptive and frequency statistics were used to determine participants' opinions about the speaking book.

Results. Results from the paired $t$-tests indicated that while a significant increase in knowledge score did not occur for all questions (no significant increase for 8/15), the overall knowledge gain was significant. Analysis of the opinion questionnaire revealed that the majority of participants had a positive reaction to the artwork, bilingual audio and text.

Conclusion. The simplicity of the narrative, the illustrations, and the bilingual text and soundtrack make the Biobanking and Me speaking book an effective tool to increase knowledge of biobanking and genetics in an easy-to-use and enjoyable way.
\end{abstract}

S Afr J Bioethics Law 2019;12(2):87-92. https://doi.org/10.7196/SAJBL.2019.v12i2.691

While biomedical research based on genetically diverse data and samples from the African continent has incredible potential to address health issues, there exists the risk of exploitative research practices, particularly when studies are conducted in environments where public knowledge of scientific concepts may be lacking. ${ }^{[1,2]}$ It is this exploitation that generates mistrust in research, and ultimately has far-reaching consequences. ${ }^{[1,3,4]}$ Increased misunderstanding and mistrust undermine research potential, and become a barrier to public participation. In medical research, the costs associated with recruitment and retention of a diversity of sample donors are not just financial. A lack of donor diversity in a research study may result in the introduction of bias, and lead to disproportionate research activities into conditions or diseases that affect specific populations. ${ }^{[5]}$ The value of creating and increasing public understanding of science through meaningful engagement platforms cannot be underestimated. For example, a study conducted on clinical trial enrolment and retention in Nigeria established that unwillingness of female respondents to participate in a clinical trial was strongly associated with low levels of awareness of and education on the clinical trials. ${ }^{[6]}$ Meaningful engagement not only increases trust in science, based on positive perceptions of social benefit, but also promotes understanding of the relevance of science and research - which is imperative when attempting to garner support from the public, and government. ${ }^{[5,7,8]}$ Positive science outreach can build strong coalitions and inspire members of the public to become champions in their communities. Science communication of broad key concepts to receptive communities becomes a gateway to more studyspecific engagement, and the active participation of stakeholders leads to capacity development and science translation. ${ }^{[1,9]}$

Recent initiatives involving the co-operation of various consortia and government departments has led to a substantial increase in the number of biobanks in Africa. In general, biobanks are referred to as 'structured collections of biological samples and associated data, stored for the purposes of present and future research.[10] Biorepositories that collect biological samples from humans can be defined as 'an organised collection of human biological material and associated data from participants, often stored for an unlimited period of time, for the purpose of health research, and managed according to professional standards under a documented governance structure.[11] The principle of a standardised biobank that serves the research community clearly points to the involvement of a diverse multitude of stakeholders, all of whom must be engaged in order to increase the legitimacy and co-operative nature of biobanks. ${ }^{[1]}$ While researchers in the biomedical field are knowledgeable regarding the technical aspects of genetic research 
and biobanking, and the ethical, legal and social implications of their scientific research, participants and community members who are the potential sample donors may find these concepts difficult to understand. ${ }^{[12,13]}$ Difficulty understanding these concepts, the technical nature of consent forms used in genetic research and the prevalence of illiteracy create a barrier to obtaining true informed consent from research participants. Engaging communities with a view to improve understanding of research principles would allow individuals to negotiate from a position of strength.

To this end, researchers at the South African (SA) National Bioinformatics Institute have developed the richly illustrated speaking book Biobanking and Me to engage individuals on topics related to genetics, biobanking and biomedical research (Fig. 1) Two completely bilingual (text and voice) versions were created, namely English-Afrikaans and English-isiXhosa. Furthermore, the speaking book has been converted into video format, and can be viewed online by following the links for the English, Afrikaans and IsiXhosa versions, respectively:

https://www.youtube.com/watch?v=5LMG4ExqlU4

https://www.youtube.com/watch?v=n8aoTkRtQ44

https://www.youtube.com/watch?v=H-r_DRYfhO8).

\section{Methods}

\section{Participant recruitment}

A total of 161 participants were recruited from a variety of nonacademic staffing sectors at the University of the Western Cape (UWC), including technical services, gardening services and cleaning services. The close proximity of the participants on the university campus itself aided in logistics for the pre- and post-test study design that was required to test the impact of the speaking book. Permission to recruit participants was obtained from the department leads for each of the service sectors. During enrolment, participant contact information was collected from those who expressed interest in the study, and participants were asked about their language preferences. In order to manage the number of interested participants, we created two groups (A and $B$ ), and split each group into language sub-groups. Identical procedure (Fig. 2) was carried out for both groups. Group A underwent assessment in the first 2-week block, and group $B$ was assessed in the second block. To control for possible cross-contamination between groups $A$ and $B$, participants were strategically recruited from different staffing sectors operating in different geographical locations on the university campus. Student research assistants fluent in isiXhosa, English and Afrikaans were involved in the recruitment, translation and assessment activities.

\section{Assessment instruments}

Two questionnaires were designed for the study. They were originally written in English, and subsequently translated into Afrikaans and isiXhosa. Bilingual research assistants assessed the translation in order to ensure accuracy. The knowledge questionnaire (KQ) was used for both the pre- and post-test to quantitatively assess participants' knowledge on topics covered in the speaking book. The first question of the $\mathrm{KQ}$ was designed to establish the willingness of the respondent to donate a biological sample. The remainder of the KQ consisted of 15 multiple-choice questions, each with four response options, of which only one option was correct. The questions were designed by the research team to cover the scope of the information in the book.

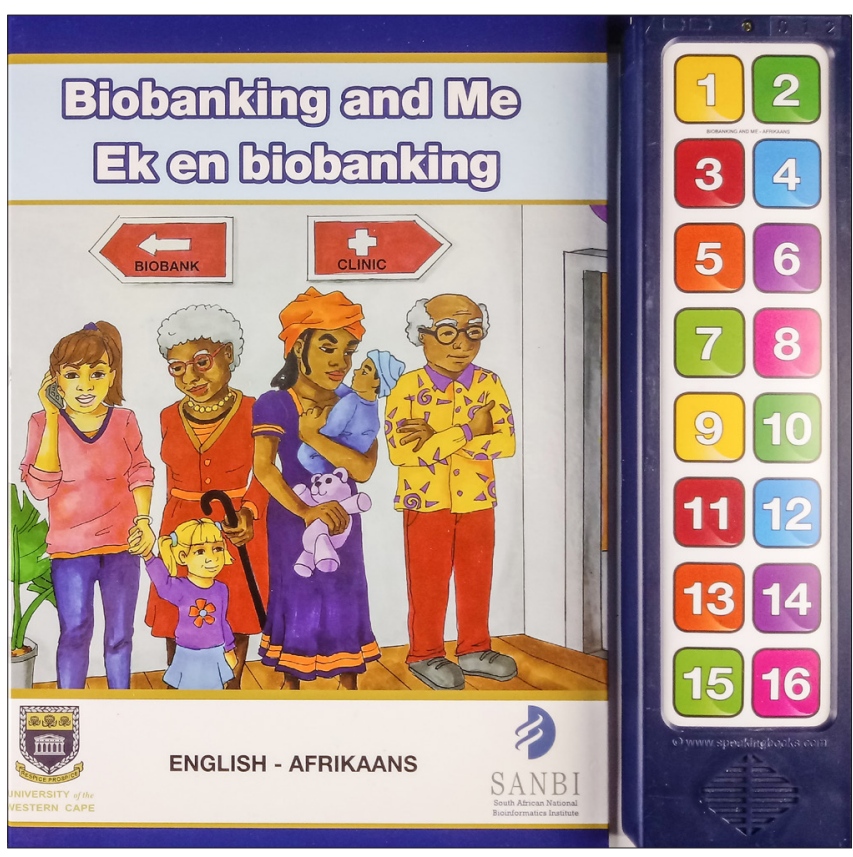

Fig. 1. The front cover of the English-Afrikaans speaking book, with a side panel of 16 buttons. Each button recites the text on the corresponding page in the book.

Participants were not informed that the pre- and post-test questions were identical.

The second questionnaire, the opinion questionnaire (OQ), was predominantly in multiple-choice format, and was used to measure the opinions of participants on various aspects of the speaking book. This questionnaire was also used to collect some demographic information. Owing to the bilingual nature of the speaking book and the monolingual nature of the assessment, only the English version of the $\mathrm{OQ}$ contained all 21 questions, while questions 2.12 and 2.13 were omitted from the isiXhosa and Afrikaans versions, respectively.

\section{Assessment}

Participants were grouped according to their language preference (English, Afrikaans or isiXhosa), as illustrated in Fig. 2. Aside from the language of the participant information sheets, consent forms, questionnaires and medium of instruction, all processes were identical for all groups. On the first day of each group's assessment, the participant information sheets and consent forms were distributed to all participants. The consent form was explained, and participants were given the opportunity to ask questions pertaining to anything they did not understand. After obtaining each participant's written informed consent, the (pre-test) KQs were distributed. Each group was asked if the questionnaires should be read out to them, and all but one group requested that the questionnaire be read. There were several assessment assistants in the room to assist participants.

Following completion of the pre-test $\mathrm{KQ}$, participants received a copy of either the English-Afrikaans speaking book or the EnglishisiXhosa speaking book, according to their preference. The speaking book served as the intervention/stimulus in this study. Operation of the book was demonstrated, and assistance was provided individually to any participant who experienced any difficulty in operating the book. Participants then took possession of the speaking book for 7 days, giving them the opportunity to read and/or listen to the content at 


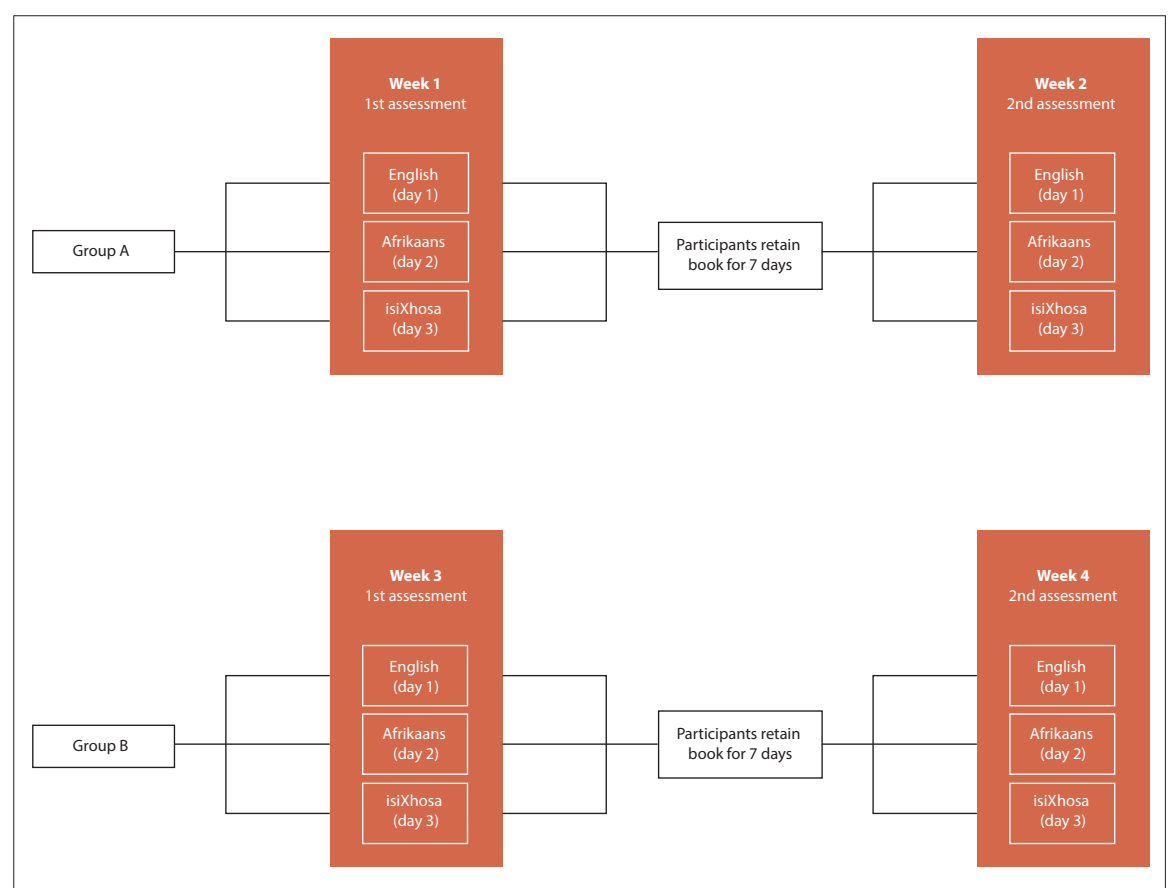

Fig. 2. Flow chart of the assessment procedure.

their own pace. Following the 7-day period, participants returned to complete the posttest KQ. In addition, during this assessment, participants were asked to complete the $\mathrm{OQ}$. Again, each group was asked if they wanted to have the questionnaire read to them. All but one group requested that the questionnaires be read to them.

\section{Data analysis}

Prior to the first assessment, and based on the recruitment forms, a unique code was assigned to each participant, and one copy of each of the questionnaires was distributed to each participant using this code. This allowed for the data to be de-identified, ensuring a higher level of participant anonymity. The results from the questionnaires were captured as three separate datasets (pre-test KQ scores, posttest KQ scores and $\mathrm{OQ}$ results) using Excel (Microsoft, USA) spreadsheets. Two different methods were used for the data analysis.

The pre-test $\mathrm{KQ}$ scores were used to establish a baseline for the level of prior knowledge of each participant on the subject matter. A correct answer was scored as 1 , and an incorrect answer as 0 . The total score for each participant was computed from their individual scores for each question. The KQ consisted of 15 items, and as such, an individual participant could score a maximum of 15 and a minimum of 0 . The post-test $\mathrm{KQ}$ was scored identically to the pre-test, and was used to gauge whether any knowledge gain had taken place after the introduction of the intervention/stimulus (the speaking book). The scores obtained from the pre-test and post-test were compared for the $\mathrm{KQ}$ as a whole, and for each individual question. These comparisons were performed using paired $t$-tests in R (R Core Team, Auckland, New Zealand) statistical software. A higher post-test KQ score when compared with the pre-test KQ score ( $p=0.05$ significance), demonstrates an improvement in knowledge on a topic.

Descriptive and frequency statistics were calculated for the $\mathrm{OQ}$ (which included questions regarding demographics and opinions, and usage of the speaking book) using SAS 9.4 (SAS, USA).

\section{Results and discussion Biographical information}

Only results and demographic information from participants who completed the study are reported. No exclusion criteria were applied to enable a respondent to participate in the study. Of the 161 participants who initially started the study, a total of 140 completed it ( $87 \%$ participant retention rate).

The distribution of male to female participants was roughly equal, with $41 \%$ of the respondents being male and 59\% female. The mean (standard deviation) age was 39 (10) years, with the youngest being 21 years old and the oldest 65 . Access of a participant to other reading material demonstrated that $29 \%$ did not have any other reading books at home. Sixty-nine percent $(N=139)$ of participants in this study had never donated a biological sample for research, and $65 \%$ percent had never heard of a biobank before $(N=139)$.

\section{Language preference and usage}

The English-Afrikaans speaking book was selected by $43 \%$ of the participants, while 57\% chose the English-isiXhosa version. Participants were able to attend assessment groups in their preferred medium of instruction, as communication in the indigenous language of the region is vital for meaningful engagement.

All participants involved in the present study received instructions and demonstrations on how to use the audio option of the book - as a result, $89 \%$ of respondents reported having listened to the book. Similarly, a study by Dhai et al. ${ }^{[3]}$ concluded that in order to maximise the impact of a speaking book as an engagement tool in waiting rooms, rural facilities and other venues, operational instructions must be included as a prerequisite to distribution.

\section{Participants' opinions of the speaking book}

Most participants felt that the speaking book was very easy to use, and $84 \%$ $(n=140)$ stated that they did not experience operational difficulty when switching between the two soundtracks. Almost all (94\%) ( $n=138)$ participants enjoyed reading and/or listening to the speaking book and indicated that they enjoyed being given the option of bilingual text (81\%) $(n=139)$ and a bilingual soundtrack (91\%) ( $n=140)$. Eightyseven percent $(n=140)$ of respondents felt that the soundtracks were audible. Almost all illustrations in the book were well-liked, as demonstrated by a cumulative percentage of $79 \%(n=140)$ of respondents indicating a positive reaction to the artwork (Fig. 3). Nearly half $(46 \%)$ of participants indicated that they understood all topics in the book, and a cumulative percentage of $97 \%$ felt that some level of learning had occurred (Fig. 4). The OQ revealed that some participants ( $2 \%$ $(n=140))$ had neither listened to, nor read, the book. Furthermore, as shown in Fig. 4, a few participants felt that they did not fully 
understand everything in the book. These results taken together may also explain why pre- and post-test scores for some questions were not found to be significant. It may be possible to refine the questionnaire to ascertain whether participants who did not read/listen to the book were also of the opinion that they did not understand everything in the book. It is worth noting that these data were obtained from the $\mathrm{OQ}$, and the feeling that a participant 'did not understand everything' is a subjective measure that may not necessarily be reflected in the individual's KQ.

Sorsdahl et al. ${ }^{[14]}$ distributed their speaking book to 213 home-based care workers who, in turn, showed the book to approximately 11000 people within a 2-week period. In another study by Dworzanowski and Wilson, ${ }^{[15]}$ their speaking book was disseminated to 7530 patients by the 193 home-based caregivers who received a copy. Similarly, results from the studies on the 'clinical trials' speaking book in SA and Uganda demonstrated that participants shared the book extensively with family, friends, neighbours and other associates, further demonstrating the value and cost-effectiveness of speaking books as an educational tool. ${ }^{[2,3]}$ The current biobank speaking book study corroborates this observation, with results indicating that most $(77 \%)$ participants shared the book with others, and would recommend the book to others (92\%). Participants in this study, on average, showed their copy of the speaking book to 4 other people, with some indicating that they shared the book with up to 20. Sharing the speaking book in this manner leads to greater dissemination within households and communities, and may indirectly contribute to its costeffectiveness of the tool.

The first question on the KQ ('Would you donate a biological sample for research?), which measured participants' opinion/ preference regarding sample donation, was specifically included to establish whether the speaking book would increase the probability of potential research participants consenting to donating a biological sample (Fig. 5). A percentage analysis comparing pre- with post-test responses indicated a $7 \%$ increase in the number of participants willing to donate a biological sample after reading and/or listening to the speaking book. The speaking book seemed to assist

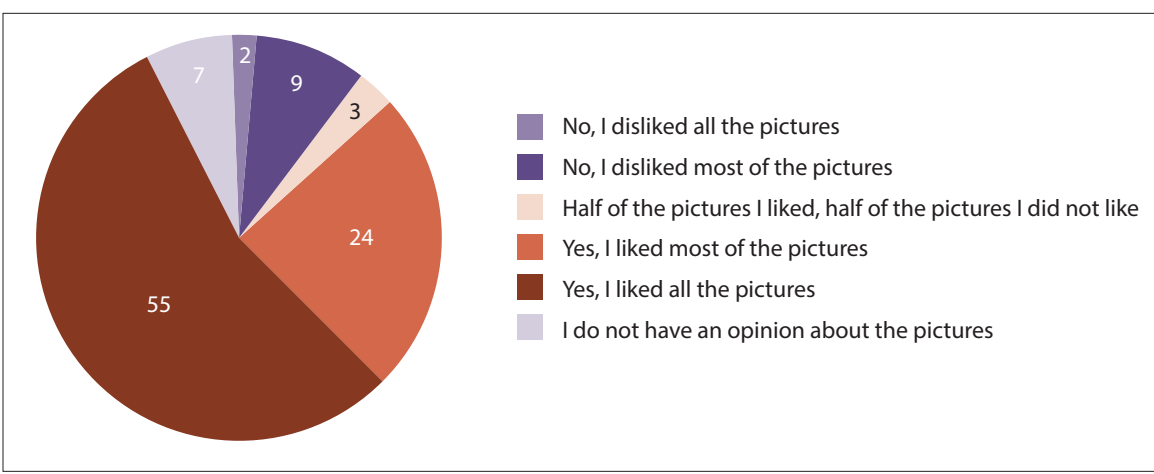

Fig. 3. Pie chart of the response percentage for the question 'Did you like the pictures in the book?' $(n=140)$.

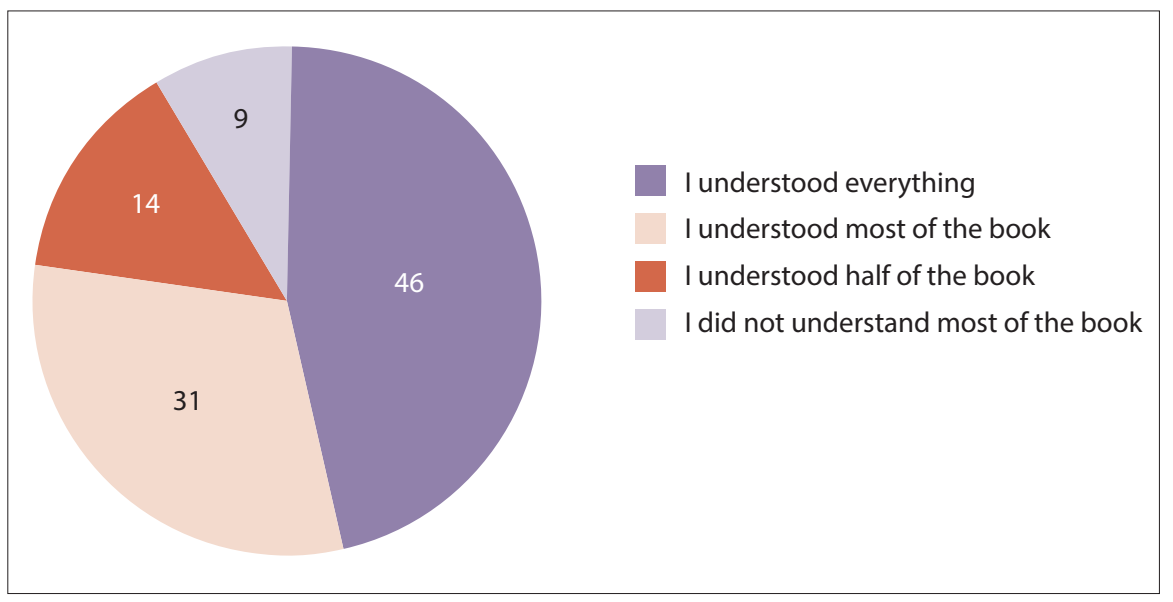

Fig. 4. Pie chart mapping response percentage for the question 'Did you understand everything in the book?' ( $\mathrm{n}=140)$.

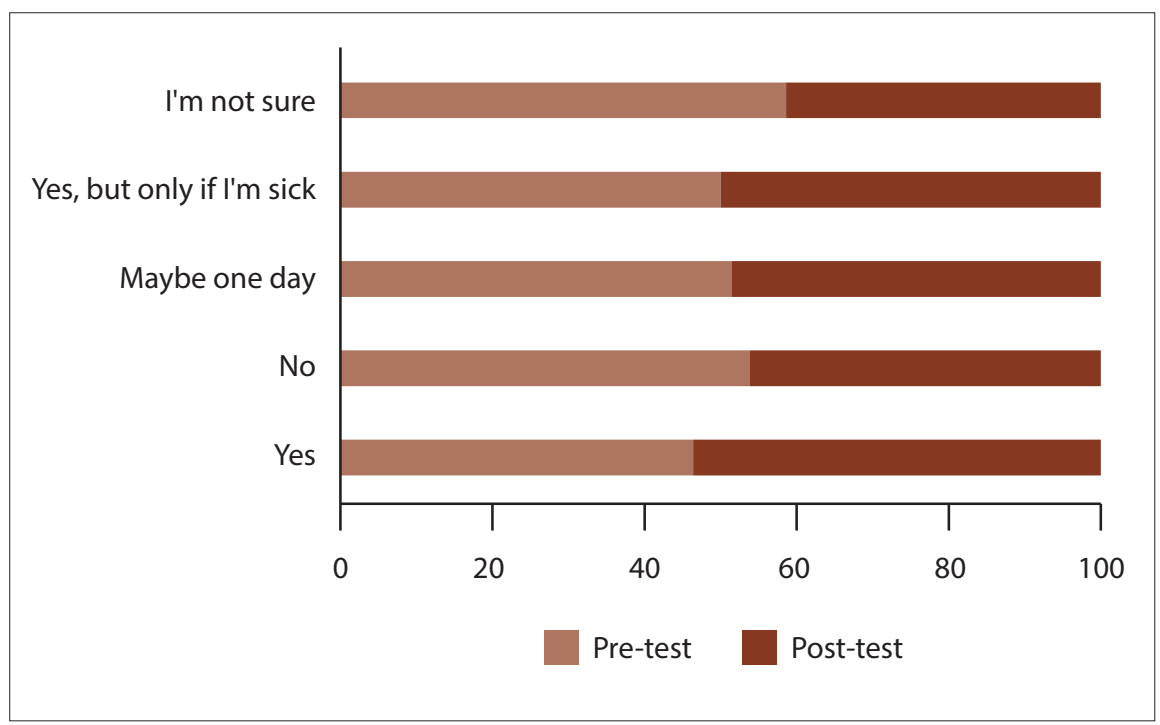

Fig. 5. Response changes to the 'Would you donate a biological sample for research?' question. (Participants who did not respond on the pre-and/or post-test were excluded; $n=104$.)

participants who were originally unsure about their decision.

\section{Knowledge gain}

Data analysis of the $\mathrm{OQ}$ coupled with the KQ demonstrated that respondents' perception that they had learned from the speaking book was accurate. Eighty percent of participants indicated in the $\mathrm{OQ}$ that they had learned a lot, while $17 \%$ said a little and $3 \%$ were unsure (Fig. 3). Several speaking books covering topics on health education 
(includingmentalhealth), disease prevention, social development and early childhood education have already been published and include both qualitative and quantitative studies on the effect of and/or people's opinion of specific speaking books, ${ }^{[2,3,14,16,19]}$ with results from these studies consistently demonstrating an overall significant gain in knowledge. Similarly, the results from the KQs in the current study indicate that while a significant increase in knowledge score did not occur for all questions (no significant increase for $8 / 15$ ), overall, knowledge gain was significant. Possible reasons for this discrepancy may have been question design (questions being too simple), or correct guessing by participants, both of which could have led to high scores being recorded in the pre-test. In a pilot study on a clinical trial speaking book, the authors surmised that baseline scores obtained by 'guesswork' may cause divergence in results. ${ }^{[3]}$ Thus if a participant guessed the correct answer in a pre-test, it would be difficult to determine whether he or she had obtained the correct answer in the post-test as a result of true knowledge gain, or of continued guessing. Significant differences between pre- and post-test scores for some questions may be explained by how a concept was explained in the book. For example, for question 2 ('Why do researchers store biological samples in a biobank?'), which showed significant difference in pre- and post- test scores $(p=0.0231)$, the text in the book makes reference to the similarity between a biobank and a library. This linkage may make the concept more relatable to participants. For question 7 ('What does DNA do?'), which did not have a significant difference in preand post- test scores $(p=0.078)$, the relevant text in the book simply states that 'DNA tells the cells what to do'. Here, a participant may not be able to frame the information in a relatable way, which could impact knowledge gain.

If this hypothesis is correct, then it provides valuable insight into how to increase the knowledge impact of future interventions by correctly contextualising complex topics. Despite this possible limitation, results obtained from analysis of the pre- and posttest scores indicate that the Biobanking and Me speaking book is an effective multimedia intervention that increases laypersons' knowledge of biobanking and genetics, and should be considered for adaptation

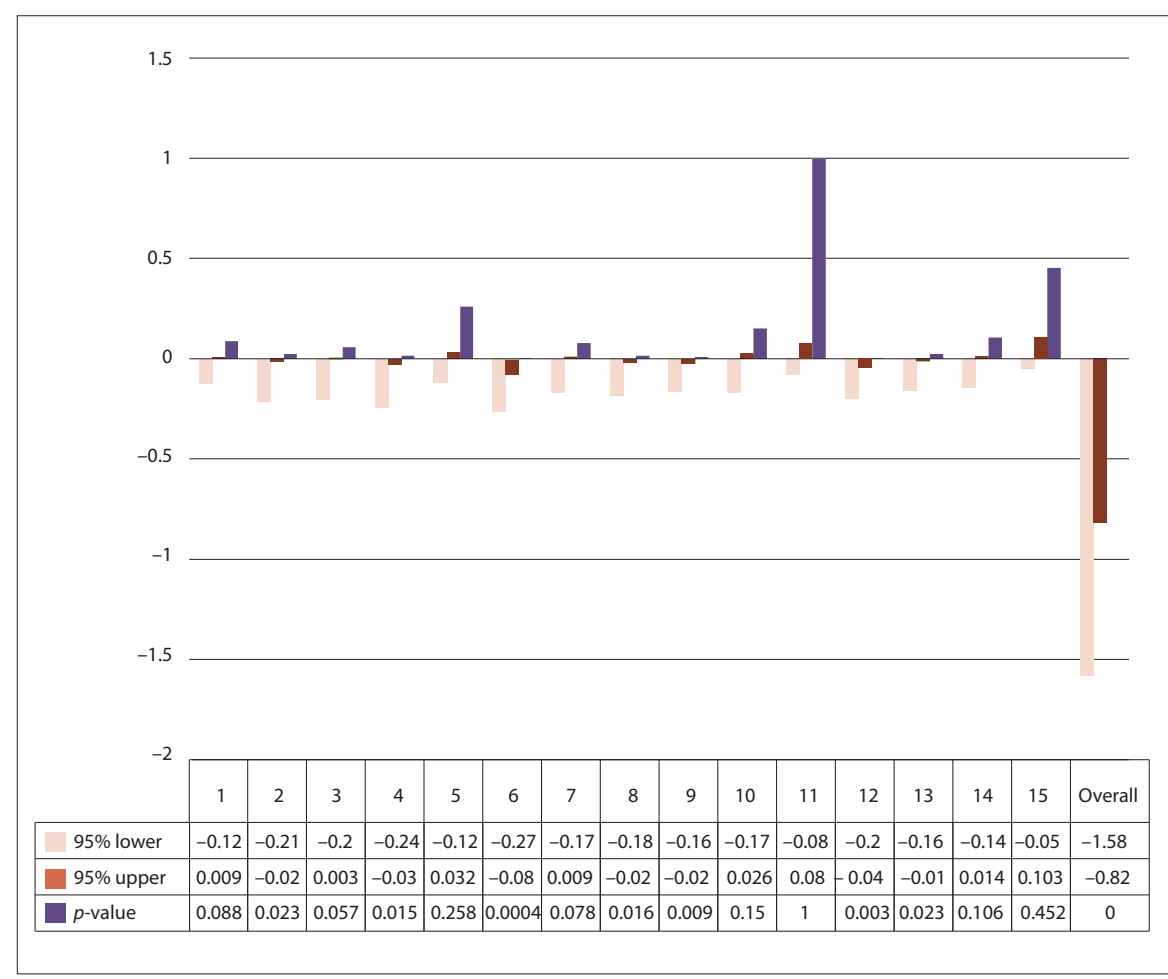

Fig. 6. Difference between pre- and post-test scores ( $\mathrm{n}=140$ ) (see supplementary file (http://sajbl.org.za/ public/sup/691.docx) for numbered questions).

into other relevant languages for wider publication and dissemination.

A negative estimated mean difference indicates that the post-test KQ score was higher than the pre-test KQ score (Fig. 6). Seven of the 15 questions saw a significant improvement in the post-test $\mathrm{KQ}$ score $(p<0.05)$. There was also a significant improvement in respondents' overall score $(p=0.000)$. Questions are found in Table 1 of the supplementary data (http://sajbl.org.za/ public/sup/691.docx).

\section{Conclusion}

The importance of genetic research and biobanks to the study of disease cannot be overstated, but huge potential for unethical and negligent practices, whether intentional or simply due to ignorance, exists. Practices that fail to protect the dignity and rights of research participants erode public trust in science, and overshadow beneficial scientific progress. Members of communities who are donors of biological samples are essential stakeholders in health research, and meaningful engagement that increases knowledge allows these donors to make informed decisions. In addition, informed donors provide a platform for public awareness, allowing communities to understand the impact of genetic research and biobanking, and may even encourage more people to donate biological samples, as demonstrated by the sharing of the speaking book by participants in this study. The simplicity of the text and explanations, the illustrations, and the bilingual text and soundtrack make the Biobanking and Me speaking book an effective tool to increase knowledge of biobanking and genetics in an easy-to-use and enjoyable way. The authors wish to encourage translation of the Biobanking and Me speaking book into other languages, and to promote increased dissemination of this resource. Finally, our results demonstrate the value of such a multisensory educational tool for public engagement, irrespective of the topic addressed. The educational nature of the speaking book empowers individuals to confidently share scientific information with others.

Acknowledgements. The authors would like to thank Hocine Bendou, Quinton Coert, Campbell Rae and Carmen Swanepoel, who assisted with the speaking book text. We would like to acknowledge all the students and staff who volunteered their time as assistants for the assessment sessions, namely, Fanechka Esterhuysen, Ziphozakhe Mashologu, Emily Stander, Lynley Adboll, Abiola Babajide, 
Mmakamohelo Direko, Susan Fernol and Fungiwe Mpithi. We also acknowledge the valuable assistance of $\mathrm{Mr}$ Jade Leon and $\mathrm{Mr}$ Peter Arendse from the Gardens and Grounds Services at UWC in arranging information sessions for us to recruit participants for the study. We thank Dr Susan Ntete from the English Department of UWC for translating the speaking book text from English to isiXhosa. We also acknowledge the funders, as well as all participants in the study.

Author contributions. $A B$ assisted in designing the project. $A B$ designed the research study, designed all questionnaires, translated all questionnaires into Afrikaans, conducted the assessments, analysed the data and wrote the first draft of the manuscript. DA contributed substantially to the initial speaking book text and study design, co-ordination of the biobank speaking book assessment workshop and the writing/editing of the article. TL contributed substantially in facilitating community engagement, the co-ordination of participants and speaking book assessment workshops. YM contributed substantially to the isiXhosa translation of the speaking book text as well as facilitating the biobank speaking book assessment workshops. RL consulted on questionnaire and experiment design, performed statistical analysis of the numerical variables measured in the experiment and compiled a report of the results and findings. RB consulted on questionnaire and experiment design, performed statistical analysis of the numerical variables measured in the experiment and compiled a report of the results and findings. AC conceived the project and contributed to its design, and assisted with the speaking book text and reviewing the artwork and audio files before production of the book. AC also provided the funding for the project and contributed to the article writing.

Funding. This project was funded by the European Union's Horizon 2020 project, B3Africa and the SA Research Chairs Initiative of the Department of Innovation and Technology and National Research Foundation of SA, award number UID 64751.

Conflicts of interest. None.

1. Moodley K. Legitimacy, trust and stakeholder engagement: Biobanking in South Africa. Asian Bioethics Rev 2017;10:325-334. https://doi.org/10.1007/s41649-017-0035-7

2. Castelnuovo B, Newell K, Manabe YC, Robertson G. Multi-media educational tool increases knowledge of clinical trials in Uganda. J Clin Res Bioeth 2014;5(1):1000165. https://doi.org/10.4172/2155-9627.1000165

3. Dhai $\mathrm{A}$, Etheredge $\mathrm{H}$, Cleaton-Jones $\mathrm{P}$. A pilot study evaluating an intervention designed to raise awareness of clinical trials among potential participants in the developing world. J Med Ethics 2010;36(4):238-242. https://doi.org/10.1136/ jme.2009.033407
4. Staunton C, Moodley K. Community engagement for biobanking research: Perspectives from Africa. Asia Pacific Biotech News 2016;20(2):14-26. https://doi. org/10.1142/S0219030316000100

5. Holzer JK, Ellis L, Merritt MW. Why we need community engagement in medical research. J Investig Med 2014;62(6):851-855. https://doi.org/10.1097/ JIM.0000000000000097

6. Chukwuneke FN, Ezeonu CT, Onyire BN, Ezeonu PO. Culture and biomedical care in Africa: The influence of culture on biomedical care in a traditional African society, Nigeria, West Africa. Niger J Med 2012;21(3):331-333.

7. Pham D. Public engagement is key for the future of science research. NPJ Sci Learn 2016;1:16010. https://doi.org/10.1038/npjscilearn.2016.10

8. Upshur RE, Lavery JV, Tindana PO. Taking tissue seriously means taking communities seriously. BMC Med Ethics 2007;8:11. https://doi.org/10.1186/14726939-8-11

9. Stilgoe J, LockSJ,Wilsdon J.Why should we promote public engagement with science? Publ Understand Sci 2014;23(1):4-15. https://doi.org/10.1177/0963662513518154

10. Parodi B. Biobanks: A definition. In: Mascalzoni D, ed. Ethics, Law and Governance of Biobanking. The International Library of Ethics, Law and Technology, vol 14. Dordrecht: Springer, 2015:15-19. https://doi. org/10.1007/978-94-017-9573-9_2

11. Pepper MS, Dandara C, de Vries J, et al. ASSAf consensus study on the ethical, legal and social implications of genetics and genomics in South Africa. S Afr J Science 2018;114(11/12). https://doi.org/10.17159/sajs. 2018/a0302

12. Tindana $P$, Campbell $M$, Marshall $P$, et al. Developing the science and methods of community engagement for genomic research and biobanking in Africa. Glob Health Epidemiol Genom 2017;2:e13. https://doi.org/10.1017/gheg.2017.9

13. Tindana PO, Singh JA, Tracy CS, et al. Grand challenges in global health: Community engagement in research in developing countries. PLOS Med 2007:4(9):e273. https://doi.org/10.1371/journal.pmed.0040273

14. Sorsdahl K, Dworzanowski B, Wilson Z. Effective intervention for depression in people living with HIV and AIDS in rural South Africa through community home-based care workers: Assessing the impact of 'speaking books'. https:// speakingbooks.com/wp-content/uploads/AIDS_depression-last.pdf (accessed 7 January 2019).

15. Dworzanowski B, Wilson Z. Exploratory reflections in pioneering a speaking book: Making mental health matter in rural South African AIDS care. https:// speakingbooks.com/wp-content/uploads/exploratory.pdf (accessed 7 January 2019).

16. Speaking books. Our library of speaking books: 2017. https://speakingbooks. com/our-library/ (accessed 7 January 2019).

17. Sorsdahl K, Stayanova R, Wilson Z, Stein D. Evaluation of SADAG's 'Suicide shouldn't be a secret' program using 'speaking books'. https://speakingbooks.com/wpcontent/uploads/Evaluation-of-S-shouldnt-be-secret.pdf (accessed 7 January 2019).

18. Dworzanowski B. 'Living with HIV and AIDS doesn't mean living with depression': An evaluation of a'speaking book' going local in peri-urban South Africa. https:// speakingbooks.com/wp-content/uploads/AIDS_depression-last.pdf (accessed 7 January 2019).

19. Endale EE, Simireta T. Rapid qualitative assessment of maternal and newborn health care (MNHC) speaking book in two districts in the Amhara Region, Ethiopia. https://speakingbooks.com/wp-content/uploads/UNICEF-RESEARCHSUMMARY-2013.pdf (accessed7 January 2019).

Accepted 14 November 2019 